\title{
Observation of an anomalous SmA-SmC-SmA phase sequence in a bent-core liquid crystal derived from 4-cyanoresorcinol
}

\author{
Yu. P. Panarin $\odot,{ }^{1,2}$ S. P. Sreenilayam $\odot,{ }^{1}$ V. Swaminathan ${ }^{\circledR},{ }^{1}$ C. Tschierske $\odot,{ }^{3}$ and J. K. Vij ${ }^{1}{ }^{\text {,* }}$ \\ ${ }^{1}$ Department of Electronic and Electrical Engineering, Trinity College, The University of Dublin, Dublin 2, Ireland \\ ${ }^{2}$ School of Electrical and Electronic Engineering, Technology University of Dublin, Dublin, Ireland \\ ${ }^{3}$ Institute of Chemistry, Organic Chemistry, Martin-Luther-University, Halle-Wittenberg, Germany
}

(Received 14 August 2019; accepted 6 December 2019; published 4 February 2020)

\begin{abstract}
The physical properties and the phase transitions of a smectic liquid crystal material built from achiral bent-core molecules are investigated by electro-optics, polarizing microscopy, birefringence, and spontaneous polarization. This material is shown to exhibit a number of orthogonal and tilted smectic phases. The observation of an anomalous phase transition on cooling from a tilted to orthogonal antiferroelectric smectic phase at lower temperatures is made, and a proof of the evidence is presented. The importance of the biaxial anchoring term in the free energy is modeled and discussed.
\end{abstract}

DOI: 10.1103/PhysRevResearch.2.013118

\section{INTRODUCTION}

Liquid crystals (LCs) belong to a unique state of soft matter where myriad liquid crystalline phases are exhibited. An emergence of these phases depends on the molecular shape, chirality, and temperature. However, the most successful LC used in the liquid crystal display (LCD) industry is still the nematic phase of rodlike (or calamitic) molecules. Calamitic LCs may also exhibit a number of smectic phases, such as orthogonal (SmA), tilted (SmC) phases, and their variants. The tilted, SmC-like smectics built from chiral molecules exhibit spontaneous polarization [1] and may form ferroelectric $\mathrm{SmC}^{*}$ and antiferroelectric $\mathrm{SmC}_{A}^{*}$ phases. A number of intermediate ferrielectric subphases may also emerge in the temperature range in between $\mathrm{SmC}_{A}^{*}$ and $\mathrm{SmC}^{*}$ [2,3]. At the time of the discovery of ferroelectric liquid crystals (FLCs), the molecular chirality was considered to be the essential condition for ferroelectricity.

Furthermore another class of LCs built from the achiral bent-core (BC) molecules exhibits not only biaxiality in a smectic phase [4] but also the polarization emerges in the bend direction from the steric interactions caused by the bent-core molecular shape inducing polar packing in layers and restricted rotations. Unlike the calamitic LCs, however the bent-core compounds even being achiral may exhibit both the spontaneous polarization and the biaxiality in orthogonal (SmA-like phases) $[5,6]$ as well as in tilted (SmC-like) smectics $[7,8]$. The common characteristics of these phases are (a) constituent molecules are achiral but (b) they possess long-range in-layer polarization and biaxiality, and these arise from the $C_{2 v}$ symmetry. The temperature dependences of both primary and secondary (i.e., biaxial) order parameters

\footnotetext{
*Corresponding author: jvij@tcd.ie

Published by the American Physical Society under the terms of the Creative Commons Attribution 4.0 International license. Further distribution of this work must maintain attribution to the author(s) and the published article's title, journal citation, and DOI.
}

in orthogonal BC phases were studied earlier $[9,10]$. The characteristics and the structures of the bent-core LC systems have been reviewed $[11,12]$.

The bent-core systems exhibit rich polymorphism in both orthogonal (SmA-like) phases [5,6] and tilted (SmC-like) phases [13-16]. The orthogonal bent-core smectic exhibits a number of mesophases such as antiferroelectric $\operatorname{SmAP}_{A}$ [6], ferroelectric $\operatorname{SmAP}_{F}$ [17], random polar $\operatorname{SmAP}_{R}$ [18], and random antiferroelectric $\operatorname{SmAP}_{A R}[19,20]$. In these achiral SmA phases, the application of electric field across the LC cell containing the aligned material causes molecular dynamics, including rotation, to occur around the long molecular axis. These systems may show promising applications in fast switching electro-optical devices [21,22].

In addition to the orthogonal SmA type phases, the bentcore LCs do show numerous additional tilted mesophases, designated as B1-B8 [23]. The simplest of these is the B2, a tilted SmC-like phase showing a large in-layer spontaneous polarization [8]. In this phase the molecules are tilted in a direction perpendicular to the bent-core plane with a $50 \%$ probability for both the left- and right-hand tilts; two chiral layers are formed with the same and opposite signs of layer chirality and tilt, leading to chiral and racemic phase structures [24-26]. Four possible structures are formed, denoted by $\mathrm{SmC}_{S / A} \mathrm{P}_{F / A}$ : synclinic/anticlinic with respect to the tilt direction and ferroelectric/antiferroelectric ordering of the spontaneous polarization vectors (see Fig. S4 in the Supplemental Material [27]) [8]. The electro-optical switching in tilted smectic phases of bent-core LCs was observed mainly in the $\mathrm{B} 2(\mathrm{SmCP})$ phase $[8,13,15,28-33]$; however, materials in this phase may not be suited for applications due to the major difficulties (a) of not achieving a good homogeneous alignment, and (b) to a coexistence of the two domains of opposite chirality.

In this paper, we report the structure and the physical characteristics of a bent-core system which shows an unusual phase sequence. We find that a reentrant orthogonal phase emerges at temperatures below the tilted smectic phases. The bent-core compound under study, $\mathbf{1} / \boldsymbol{n}=\mathbf{1} / \mathbf{1 6}$ (PAL29), is a member of the homologous series of 4-cyanoresorcinol 


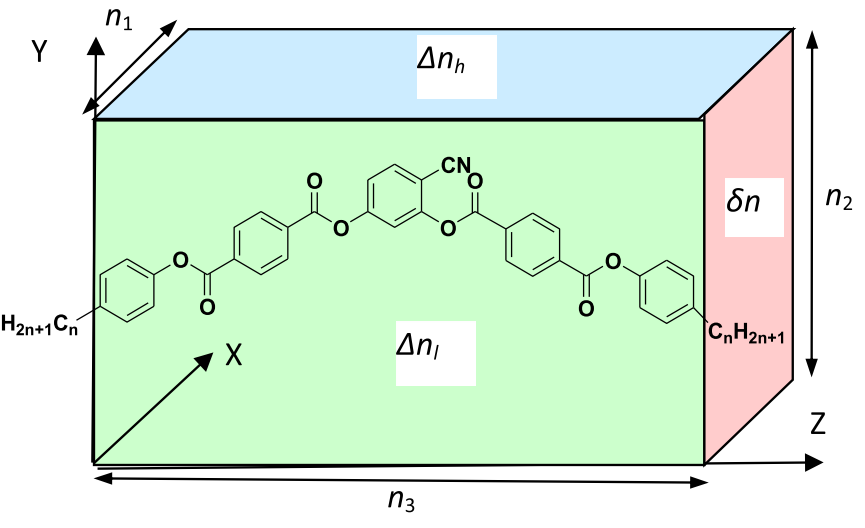

FIG. 1. Molecular structure of the homologous series of 4cyanoresorcinol bisbenzoate with two terephthalate-based wings and the biaxial model; the compound under discussion here is $1 / \boldsymbol{n}=$ 1/16. Refractive indices in three directions; their magnitudes are $n_{1}<n_{2}<n_{3} . \Delta n_{l}=n_{3}-n_{2} ; \Delta n_{h}=n_{3}-n_{1}$, and $\delta n=n_{2}-n_{1}$. The subscripts $l$ and $h$ correspond to low and high values of birefringence.

bisbenzoates with two terephthalate-based wings terminated by identical linear $n$-alkyl chains on both ends. The molecular structure of this series is shown in Fig. 1. The unique feature of compounds $\mathbf{1} / \boldsymbol{n}$ is a relatively small molecular bend ( $\alpha=\sim 140^{\circ} ; \alpha$ is the angle between the two rodlike wings of the bent-core mesogens) compared to typical bentcore mesogens $\left(\alpha=\sim 120^{\circ}\right)$, providing the unique properties of these compounds. Mainly it supports the formation of orthogonal and weakly tilted $\mathrm{SmC}$ phases with randomized (de Vries-like), heliconical, and anticlinic tilt correlations. Moreover, their lamellar phases do not show any indication of a tilted organization of the molecules in their x-ray diffraction patterns, either in the development of the $d$ spacing at the SmA-SmC transition, or in the two-dimensional (2D) diffraction patterns of the aligned samples, where the layer reflection is perpendicular to the diffuse wide angle scattering (Figs. S1 and S2 in the Supplemental Material [27]) [34]. Therefore, based on the x-ray scattering, we initially identified nontilted smectic phases. Later it was recognized for $\mathbf{1} / \boldsymbol{n}=\mathbf{1} / \mathbf{1 6}$ [34] and for $\mathbf{1} / \boldsymbol{n}=\mathbf{1} / \mathbf{1 4}$ [35] that the molecular organization in the polar smectic phases is actually tilted. Table S1 in the Supplemental Material [27] gives an overview of our present understanding of the phase sequence of 1/16. When cooling from the isotropic state, it transforms to an orthogonal SmAlike phase $[9,10,12]$ at higher temperatures with a series of paraelectric $\left(\mathrm{SmCP}_{R}\right)$ and polar tilted (B2-like) SmC phases $\left(\mathrm{SmC}_{S} \mathrm{P}_{F}^{\text {hel }}, \mathrm{SmC}_{A} \mathrm{P}_{A}\right)$ exhibited at lower temperatures $[34,36]$. The most fascinating feature of these long chain compounds is the formation of a heliconical phase designated as $\operatorname{SmC}_{S} \mathrm{P}_{F}^{h e l}$, identified first by the observation of a helical structure in planar-aligned samples of 1/16 and 1/18, by AFM of 1/16 [34], and these were unambiguously confirmed in $\mathbf{1} / \mathbf{1 4}$ by soft resonant $\mathrm{x}$-ray scattering [35]. The superscript hel in $\mathrm{SmC}_{S} \mathrm{P}_{F}^{\text {hel }}$ was used in [34] to stress the existence of a helix in this LC phase formed by achiral molecules. The helix axis is perpendicular to the layers plane; these are composed of synclinic and polar ordered molecules $\left(\mathrm{SmC}_{S} P_{F}\right.$ layers $)$. This phase has also been designated as $\mathrm{SmCP}_{\alpha}$ [37] or $\mathrm{Sm}(\mathrm{CP})_{\alpha}$ [35] because of its analogy to the $\mathrm{SmC}_{\alpha}^{*}$ phases formed by chiral rodlike molecules $[2,3]$.
It is reported herein that the compound under study actually shows the following phase sequence: Iso $162^{\circ} \mathrm{C} \mathrm{SmA} 132^{\circ} \mathrm{C}$ $\mathrm{SmCP}_{R} 110^{\circ} \mathrm{C} \mathrm{SmC}_{S} \mathrm{P}_{F}^{\text {hel }} 92^{\circ} \mathrm{C} \mathrm{SmC}_{A} \mathrm{P}_{A} 82^{\circ} \mathrm{C} \mathrm{SmAP}_{A} 77^{\circ} \mathrm{C}$ $\mathrm{Cr}$ (for phase abbreviations, see Table $\mathrm{S} 1$ of the Supplemental Material [27]). We find that there is an additional, previously not recognized, low-temperature phase $\operatorname{SmAP}_{A}$ which is a nontilted smectic phase occurring in an inverted phase sequence below the tilted one. Moreover, it is also shown that the $\mathrm{SmC}_{A} \mathrm{P}_{A}$ phase between $\mathrm{SmC}_{S} \mathrm{P}_{F}^{\text {hel }}$ and $\mathrm{SmAP}_{A}$ is unusual, as it forms a unique surface-stabilized (SS) structure with the optical axis directed parallel to the smectic layer normal.

\section{EXPERIMENTAL METHODS AND RESULTS}

The planar cells used to study the electro-optic response are constructed by spin-coating the polymer solution RN 1175 (Nissan Chemicals, Japan) on the inside surfaces of the two substrates; these are polymerized by baking at a temperature of $250^{\circ} \mathrm{C}$ for $1 \mathrm{~h}$. The surfaces are rubbed using a commercial rubbing machine. Homeotropic cells are constructed from substrates whose surfaces are coated by the polymer solution AL60702 (JSR, Korea), the solution polymerized to form a thin alignment layer. The ITO electrodes on the lower substrate are etched with a gap of $80 \mu \mathrm{m}$ to produce a lateral electric field. The cell thickness is controlled by Mylar spacers of different thicknesses. This is measured using the technique of optical interference. The LC samples are studied using a polarizing optical microscope (Olympus BX 52) equipped with an Instec hot stage, connected in turn to a Eurotherm 2604 temperature controller that allows for a temperature stability of the sample to within $\pm 0.02{ }^{\circ} \mathrm{C}$.

The molecular tilt angle is measured by two independent techniques. In the first one, it is measured as half the rotation angle, between the positions of the maximal extinctions for $\pm E$. This method when used manually is rather crude and time consuming and has only a limited measurement accuracy of $\pm 1^{\circ}$ in the tilt angle. Here the hot stage is fixed on the rotation table of the polarizing optical microscopy (OPM); the rotation of the table is automated by a motor. The second technique [38] is based on monitoring the electro-optical response of a LC cell inserted in between the crossed polarizers. The optical axis of the cell (i.e., the layer normal) is fixed to lie at an angle of $22.5^{\circ}$ to one of the two directions of the crossed polarizers. Here the optical response is simply proportional to the induced or the switching angle in the small angle approximation. This method gives a higher accuracy in the tilt angle. This technique is easily automated; however, the maximal tilt angle is restricted to $22.5^{\circ}$. Both methods give practically identical values of the optical tilt angle. Figure 2(a) shows the temperature dependence of the tilt angle measured by using $60 \mathrm{~V}$ peak to peak amplitude of the square voltage across the cell in the first method $(0.5 \mathrm{~Hz}$; data points shown as open squares), while for the second method $(f=30 \mathrm{~Hz}$, the data are shown by filled circles). The most remarkable result is the observation of a reversed temperature dependence of the tilt angle in the tilted smectic phases. The angle after having reached the maximum gradually decreases and finally the structure transforms to an orthogonal SmA-like phase. Such an inverted phase sequence, SmC-SmA, was observed on cooling only in the case of a class of dimesogens with odd spacers [39]. In that case, both smectic phases were apolar, 

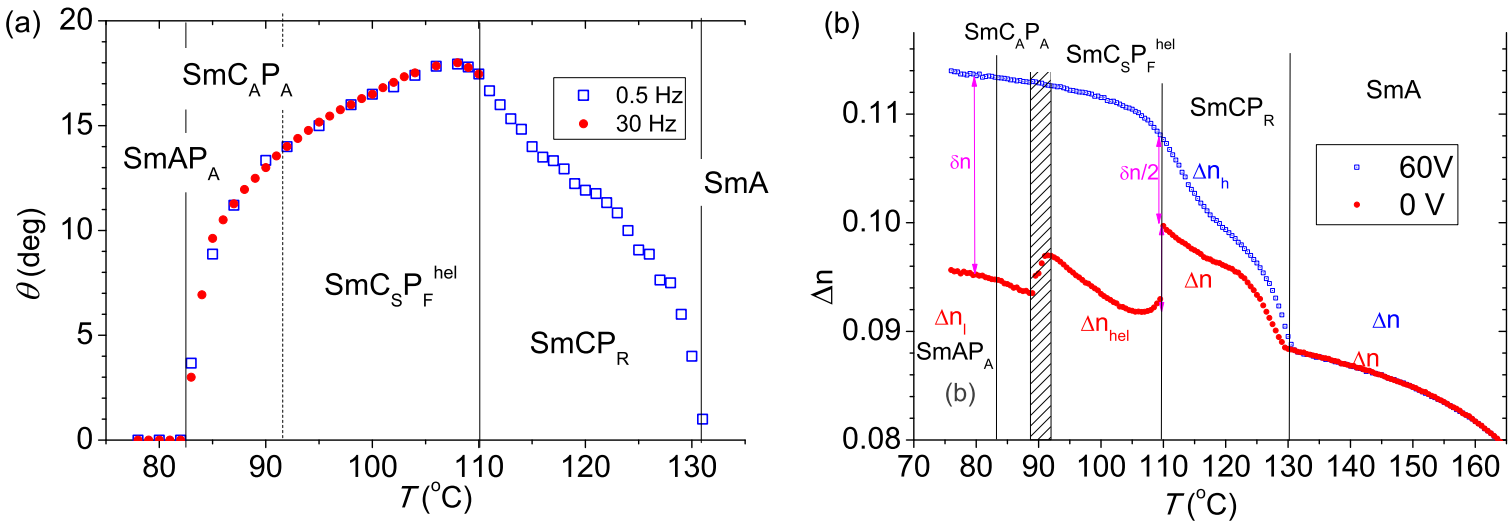

FIG. 2. (a) Plots of the temperature dependence of the optical tilt angle. Blue open squares in (a) correspond to the tilt angle measured by applying a square wave_- peak-to-peak amplitude, $60 \mathrm{~V}$, frequency $0.5 \mathrm{~Hz}$, by the method of finding half the angle between the two consecutive extinction positions. Red filled circles correspond to the measurements made for a peak-to-peak amplitude of $60 \mathrm{~V}$, frequency $30 \mathrm{~Hz}$ using the second automated method (see text). In (b), the measured effective birefringence values of a planar-aligned LC cell values for a wavelength $\lambda=550 \mathrm{~nm}$, using the method given in [40], are plotted as a function of temperature. The refractive index increments are defined in the caption of Fig. 1. Red filled circles in (b) correspond to the birefringence measured in the absence of the applied field and blue open circles correspond to the measured values in the presence of the electric field, square wave peak-to-peak amplitude: $60 \mathrm{~V}, f=30 \mathrm{~Hz}$. The striped region at a temperature of $90^{\circ} \mathrm{C}$ corresponds to the coexistence of (i) the helical phase, and (ii) the surface-stabilized $\mathrm{SmC}_{A} \mathrm{P}_{A}$ structure.

while the studied material here is a bent-core mesogen and has two orthogonal phases, a high-temperature paraelectric SmA phase and a polar low-temperature $\operatorname{SmAP}_{A}$ phase separated by a polar heliconical $\mathrm{SmC}$ phase $\left(\mathrm{SmC}_{S} \mathrm{P}_{F}^{\text {hel }}\right)$, a uniquely interesting observation.

The birefringence measurements are based on the spectral study of the transmitted light through a liquid crystal cell placed between the crossed polarizers in which the optical transmittance spectra are fitted using the technique described in Ref. [40]. Results of these fitting give wavelength dispersions of the birefringence as a function of temperature and of applied voltage. Figure 2(b) shows the plot of birefringence measured for $\lambda=550 \mathrm{~nm}$ as a function of temperature after having been corrected for its dispersion with wavelength. Figure 2(a) presents results of the temperature dependence of the optical tilt angle for a 9- $\mu \mathrm{m}$ planar-aligned cell measured with $(60 \mathrm{~V})$ applied voltage at frequencies of 0.5 and $30 \mathrm{~Hz}$, both under cooling. The temperature dependencies of the tilt and birefringence, $\theta(\mathrm{T})$ and $\Delta n(\mathrm{~T})$, show unusually interesting and complicated dependencies on temperature. We surmise that the optical tilt angle is a measure of the tilt angle of the mesogen, whereas the x-ray tilt angle corresponds to the scattering from the entire molecule and hence its value is close to zero and lies within an experimental error of $\pm 3^{\circ}$ [34]. For details see the Supplemental Material [27].

\section{DISCUSSION}

The anisotropic properties of LCs can be characterized by the molecular polarizability tensor $\chi_{n, i j}^{M}$, diagonalized in the molecular frame as

$$
\chi_{n, i j}^{M}=\chi_{33} a_{i} a_{j}+\chi_{22} b_{i} b_{j}+\chi_{11} c_{i} c_{j},
$$

where the orthogonal unit vectors $\boldsymbol{a}, \boldsymbol{b}$, and $\boldsymbol{c}$ are the principal molecular axes and $\chi_{11}, \chi_{22}$, and $\chi_{33}$ are the principal polarizability values. $M$ stands for molecular/microscopic, $n$ refers to the $n$th molecule.
The components of average polarizability depend on the orientational order parameter and these are conveniently calculated by rewriting the average polarizability tensor as a sum of the orthogonal contributions:

$$
\chi_{i j}=\left\langle\chi_{n, i j}^{M}\right\rangle=\bar{\chi} \delta_{i j}+\Delta \chi\left\langle\left(a_{i} a_{j}-\frac{1}{3} \delta_{i j}\right)\right\rangle+\chi_{\perp}\left\langle\left(b_{i} b_{j}-c_{i} c_{j}\right)\right\rangle .
$$

where $\bar{\chi}=\left(\chi_{11}+\chi_{22}+\chi_{33}\right) / 3$ is the average molecular polarizability, $\Delta \chi=\chi_{33}-\left(\chi_{11}+\chi_{22}\right) / 2$ is the polarizability anisotropy, and $\chi_{\perp}=\chi_{22}-\chi_{11}$ is the biaxiality of molecular polarizability; $\langle(\cdots)\rangle$ denotes the statistical average.

Based on the polarizability tensor, one can establish a second rank refractive index tensor $\left\langle n_{i}\right\rangle$, elements of which are calculated from a set of equations, $n_{i}=\sqrt{1+4 \pi \chi_{i i}}$. We fix $n_{3}$ as the refractive index along the long molecular axis, $n_{2}$ as along the bend direction, and $n_{1}$ perpendicular to both $n_{2}$ and $n_{3}$. These are shown in Fig. 1. On assuming $n_{1}<$ $n_{2}<n_{3}$, one introduces the biaxiality, $\delta n=n_{2}-n_{1}$, and the two birefringence terms, $\Delta n_{h}=n_{3}-n_{1}$ (high) and $\Delta n_{l}=$ $n_{3}-n_{2}$ (low) for the biaxial phase.

In uniaxial $\mathrm{SmA}$ and $\mathrm{SmCP}_{R}$ phases, where the free molecular rotations around the long molecular axis are plausible, one can introduce the refractive index in a plane perpendicular to the long molecular axis $n_{\perp}=\frac{n_{1}+n_{2}}{2}$ and the average birefringence $\Delta n=n_{3}-n_{\perp}$ with the following relations: $\delta n<\Delta n_{l}<\Delta n<\Delta n_{h} ; \Delta n_{h}-\Delta n=\Delta n-\Delta n_{l}=$ $\delta n / 2 ; \Delta n_{h}-\Delta n_{l}=\delta n$.

The temperature dependence of the birefringence is measured with and without applied voltage. In the temperature range $160{ }^{\circ} \mathrm{C}-132{ }^{\circ} \mathrm{C}(\mathrm{SmA})$, birefringence with field is almost the same as without field [Fig. 2(b)]. The polarizing optical microscopy (POM) of planar cells [34] shows that textures in this temperature range appear similar to that of a conventional uniaxial SmA phase, with the optical axis directed along the rubbing direction $\boldsymbol{R}$. This is supported by the perfect extinction seen in homeotropic texture, due to 
the orthogonal molecular organization in layers [34]. In this temperature range, the data in general are typical of the SmA and the unusual and the intriguing phenomena begin at the phase transition to the tilted phase.

On further cooling the cell to the phase transition to the tilted $\mathrm{SmCP}_{R}$ phase $[34,36]$ at $\sim 132{ }^{\circ} \mathrm{C}$, the uniform planar texture under the applied field breaks into the two sets of domains with their optical axes directed at $\pm \theta$ to the rubbing direction; $\theta$ is the temperature-dependent tilt angle [Fig. 2(a)]. The homeotropic textures show a typical schlieren texture due to the molecular tilt angle being finite [34]. In the planaraligned samples, initially, both the birefringence and the tilt angle increase with decreasing slope as the temperature is reduced. This is typical of the SmA-SmC type phase transition. Then at temperatures within $\sim 120{ }^{\circ} \mathrm{C}-115^{\circ} \mathrm{C}$, both tilt angle and the birefringence continue to increase with a higher rate/slope than before.

Another feature of the observations is an increase in the birefringence on the application of the electric field. One may explain a small increase arising from the effect of alignment by the applied field; this normally improves the quality of the texture and hence may increase the birefringence somewhat, but such a large increase $(\sim 10 \%)$ is hard to explain. On the contrary, it can be explained as follows: The $\mathrm{SmCP}_{R}$ phase is a tilted paraelectric phase where the biaxial-shaped molecules rotate around the long molecular axis. As a consequence, in the absence of applied field, the resulting birefringence is the average value $\Delta n=n_{3}-n_{\perp}$ as in a uniaxial phase. An application of external electric field deforms the uniform/random rotations of the molecules and aligns the molecules perpendicular to the electric field increasing the birefringence from $\Delta n$ to $\Delta n_{h}$. This effect is stronger at lower temperatures with an increase in the electric field and hence the difference in the birefringence with and without field $\Delta n_{h}-\Delta n$ should be up to $\delta n / 2$. The biaxiality of the compounds of the same homologous series $\mathbf{1} / \boldsymbol{n}=\mathbf{1} / \mathbf{8}, \mathbf{1} / \mathbf{1 2}, \mathbf{1} / \mathbf{1 4}, \mathbf{1} / \mathbf{1 8}$ was estimated as $\sim 0.015-0.02[9,41]$. This is in good agreement with the experiment result [Fig. 2(b)]; i.e. at a temperature of $110^{\circ} \mathrm{C}$ $\Delta n_{h}-\Delta n=0.108-0.100=0.008 \approx \delta n / 2$.

On cooling the cell below a temperature of $110^{\circ} \mathrm{C}$, the field-induced two-domain texture of the planar-aligned cell shows the transition to a uniaxial texture like that of SmA but the birefringence value without the applied field suddenly drops from $\sim 0.11$ to $\sim 0.093$. However, the birefringence with applied field continues to grow on cooling. This phenomenon is well explained arising from the spontaneous formation of a helical structure in the $\mathrm{SmC}_{S} \mathrm{P}_{F}^{\text {hel }}$ phase [34]. Recently, a short helix (of $\sim 2.8$ smectic layers) was confirmed to exist by resonant soft x-ray scattering (RSXS) in a shorter compound of the homologous series $(\mathbf{1} / \mathbf{1 4})$. Normally the helix formation results from molecular chirality of the compound but here the molecule is achiral and the helix here is formed as a result of first achiral symmetry breaking taking place and then resulting from a minimization of the electrostatic energy where the spontaneous in-layer polarization [42] is significantly reduced. It is known that the effective birefringence of a helical structure is lower than of the flat planar-aligned structure by a factor $\Delta n_{h e l}(\theta)=\frac{\Delta n}{2}\left(3 \cos ^{2} \theta-1\right)$. This difference is used in calculating the molecular tilt angle in the undisturbed helix or vice versa. On knowing the tilt angle, we can then estimate an effective birefringence of the helical structure. On using this formula, and using $\Delta n=0.105$ and $\theta=17^{\circ}$, we obtain $\Delta n_{h e l} \approx 0.092$, which again is in good agreement with the experimental result.

We investigate the electro-optical properties of the $\mathrm{SmC}_{S} \mathrm{P}_{F}^{\text {hel }}$ phase. An application of the external electric field disturbs the helical structure, which leads to an emergence of the macroscopic tilt angle. This may be called the linear electro-optic effect, similar to that of the deformed helix electro-optical effect observed in conventional FLCs (the socalled DHFLC effect) [43]. This effect for an achiral bent-core system has been studied in detail in [44] and it exhibits a fast electro-optical response with a tunable analog gray scale. Figure 3(a) presents the temperature dependence of the electrooptical response of a planar-aligned $8-\mu \mathrm{m}$ cell placed between the crossed polarizers where the smectic layer normal (optical axis) makes an angle of $22.5^{\circ}$ to the polarizer/analyzer direction. In this geometry, the first harmonic of the electro-optical response is simply proportional to the induced or the switching angle, in the small angle approximation. In the $\operatorname{SmC}_{S} \mathrm{P}_{F}^{\text {hel }}$ phase, the electro-optical amplitude is almost linearly proportional to the applied electric field as in the DHFLC. On the
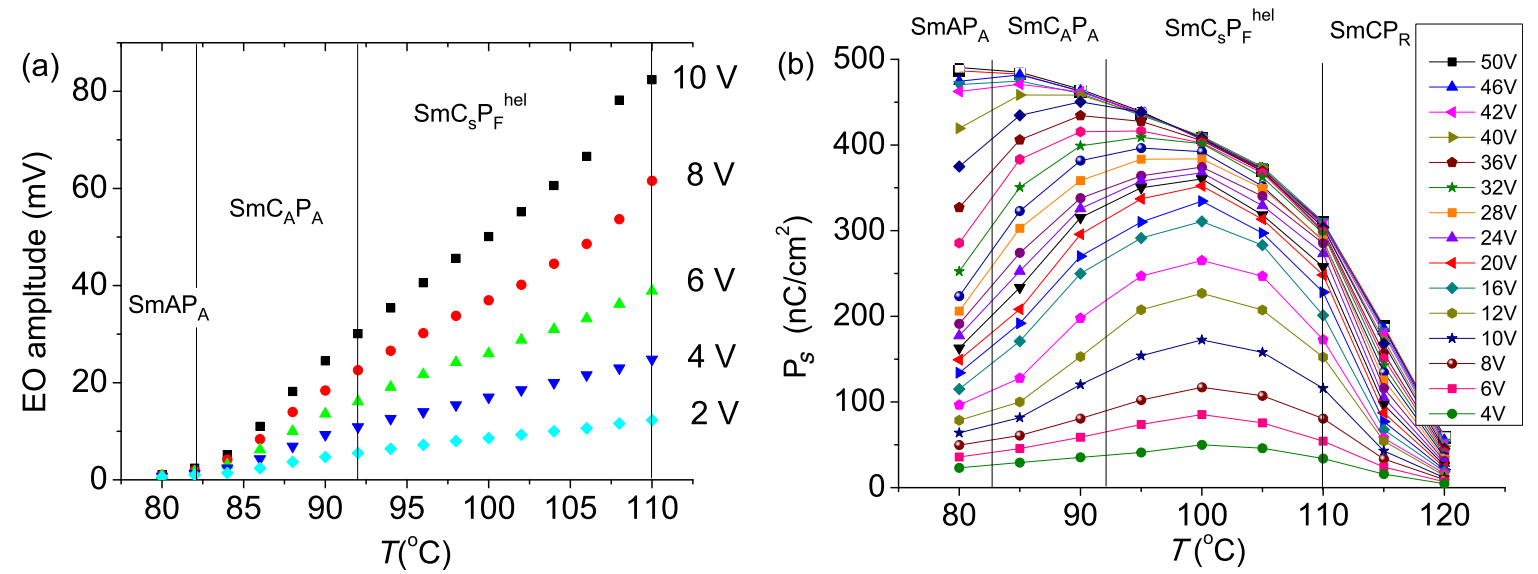

FIG. 3. Temperature dependence of (a) the amplitude of first harmonic of the EO response and (b) the macroscopic spontaneous polarization of $\mathbf{1} / \mathbf{1 6}$ for different applied voltages. The output of the photodetector is in $\mathrm{mV}$; the ordinate scale in Fig. 3(a) is in arbitrary units. 
other hand, the electro-optical amplitude gradually decreases under cooling from the maximal value at $110^{\circ} \mathrm{C}$, where the tilt angle is close to a maximum value of $\theta \sim 18^{\circ}$, down to a temperature of $82^{\circ} \mathrm{C}$ where the tilt angle does drop to zero and the structure of the phase becomes orthogonal [Fig. 2(a)]; hence it does not show a linear electro-optical response.

In the $\mathrm{SmC}_{S} \mathrm{P}_{F}^{\text {hel }}$ phase, the birefringence after the initial drop grows on cooling with increasing rate. This can be explained by the temperature dependence of the tilt angle. The molecular tilt angle reaches the maximal value at the phase transition temperature of $110^{\circ} \mathrm{C}$. Then, surprisingly, unlike the other tilted smectic phases, it begins to decrease on cooling. Furthermore at a temperature of $\sim 82^{\circ} \mathrm{C}$, the tilt angle reaches zero. In other words, a phase transition occurs from the tilted to the orthogonal smectic phase. Figure 3(b) shows results of the temperature dependence of the macroscopic spontaneous polarization $\left(P_{\mathrm{S}}\right)$ at different applied voltages. The temperature dependence of $P_{\mathrm{S}}$ proportional to the $\mathrm{EO}$ amplitude [Fig. 3(a)] shows a strong dependence on the applied voltage. At voltages below $\sim 40 \mathrm{~V}$, the helical structure may still persist in the cell; hence the measured $P_{\mathrm{S}}$ is lower than the maximum attainable spontaneous polarization. In this field range the amplitude of the EO response and hence of $P_{\mathrm{S}}$ [Fig. 3(b)] is initially proportional to the applied field arising from the field-induced helical distortion. Then it continues to grow at a decreasing slope until it saturates at the threshold field corresponding to the helix unwinding. The threshold field (voltage/cell thickness) is also temperature dependent; it grows on cooling from $\sim 30 \mathrm{~V}\left(110^{\circ} \mathrm{C}\right)$ to $\sim 50 \mathrm{~V}$ (at $80^{\circ} \mathrm{C}$ ).

According to the experimental results discussed above for the phase below $82^{\circ} \mathrm{C}$, the optical axis is parallel to the smectic layer normal and/or the rubbing direction. In the absence of the electric field, the structure is helix free; i.e., all of the molecules lie parallel to the substrates resulting in the lowest birefringence value, $\Delta n_{l}$. The application of an electric field higher than the threshold turns the molecular planes in the direction of the electric field and perpendicular to the surface plane, resulting in the higher birefringence value, $\Delta n_{h}$, as is observed. This is in good agreement with the experiment on finding that $\Delta n_{h}-\Delta n_{l}=\delta n$, as shown in Fig. 2(b).

Hence such a structure may be assigned either to an orthogonal antiferroelectric or ferroelectric SmA-like phase, i.e., $\mathrm{SmAP}_{A}$ or $\mathrm{SmAP}_{F}$. Optically both phases are equivalent to each other since the primary optical axis in both is always parallel to the smectic layer normal. This also explains why the electro-optic response due to the reorientation of the optical axis by the field is absent. Instead, the switching here is due to the secondary director. A change in the color is due to an increase in the effective birefringence (from $\Delta n_{l}$ to $\Delta n_{h}$ ) as discussed above. Therefore, the data presented in Figs. 2 and 3 are not sufficient to favor the antiferroelectric phase over the ferroelectric one.

In a conventional AFLC, this ambiguity can be resolved by studying the temperature and voltage dependencies of $P_{\mathrm{S}}$ shown in Fig. 3(b). The antiferroelectric phase normally shows tristable switching which leads to a double hysteresis loop. Ferroelectric LCs show a single hysteresis loop or continuous switching. In our case, the spontaneous polarization curves [Fig. 3(b)] evolve continuously across the phase transition at $82^{\circ} \mathrm{C}$ without showing any specific behavior typical of the ferroelectric to antiferroelectric phase transition. However, the orthogonal $\mathrm{SmAP}_{A}$ materials also exhibit a large continuous pretransitional effect $[6,45,46]$. For this phase, an electric field of $3-4 \mathrm{~V} / \mu \mathrm{m},(\sim 30-40 \mathrm{~V}$ for a $9-\mu \mathrm{m}$ planaraligned cell) usually saturates $P_{\mathrm{S}}$. This is in good agreement with our results [Fig. 3(b)].

An antiferroelectric $\mathrm{SmAP}_{A}$ phase rather than a ferroelectric $\mathrm{SmAP}_{F}$ in the ground state is more likely to occur at lower temperatures. Several reasons for this assignment can be given. The free energy of $\mathrm{SmAP}_{A}$ is lower than that of $\mathrm{SmAP}_{F}$, where the large in-layer spontaneous polarization makes the ferroelectric ordering energetically unstable. Most bent-core SmAP materials are antiferroelectric; i.e., $\operatorname{SmAP}_{A}$ exists preferably over $\operatorname{SmAP}_{F}$ at lower temperatures. The only exception to this rule is the existence of the $\operatorname{SmAP}_{F}$ phase observed in [17]. The compound in [17] has a specific siloxane tail which usually stabilizes de Vries SmA and this may also stabilize the ferroelectric structure as well. The assignment is also confirmed by the free-energy calculations. These indicate that an antipolar smectic $\mathrm{A}\left(\mathrm{SmAP}_{A}\right)$ phase is more stable than a polar smectic $\mathrm{A}$ phase $\left(\mathrm{SmAP}_{F}\right)$ due to a reduction in (a) the electrostatic free energy and (b) the entropy associated with the out-of-layer fluctuations [47].

Based on the observations and the ensuing discussion given here, we conclude that the phase below a temperature of $82^{\circ} \mathrm{C}$ is the antiferroelectric orthogonal smectic A type phase, similar (or identical) to the $\mathrm{SmAP}_{A}$ phase observed in various orthogonal smectic bent-core LCs at higher temperatures. The existence of a double-layer periodicity in the low-temperature phase was observed by carbon-edge resonant soft RSXS in the shorter 1/14 compound [35]. In contrast to the other reported $\mathrm{SmAP}_{A}$ phases, this orthogonal phase is observed in the temperature range below to those of the tilted smectic phases.

Another interesting feature of the studied material is as follows: The temperature dependencies of the tilt angle [Fig. 2(a)] and of the amplitude of the electro-optical response [Fig. 3(a)] clearly show that a phase transition occurs from the tilted to the orthogonal smectic phase at $\sim 82^{\circ} \mathrm{C}$; nevertheless the temperature dependence of the birefringence in the absence of the field shows a smooth drop from $92^{\circ} \mathrm{C}$ to $89^{\circ} \mathrm{C}$ and the birefringence changes continuously on passing the phase transition temperature $\sim 82^{\circ} \mathrm{C}$. To explain such a behavior of the birefringence data we note that its value at temperatures above $92^{\circ} \mathrm{C}$ corresponds to the helical structure while in the temperature range below $89^{\circ} \mathrm{C}$ it equals $\Delta n_{l}$, where the molecules lie flat on the surface plane. Therefore, at $\sim 92^{\circ} \mathrm{C}$ the helical structure gradually transforms to a surfacestabilized (SS) structure with the optical axis directed parallel to the smectic layer normal. In the intermediate range between $89^{\circ} \mathrm{C}$ and $92^{\circ} \mathrm{C}$ both structures coexist. This scenario is in good agreement with the resonant XRD study [35] of $\mathbf{1} / \boldsymbol{n}=\mathbf{1} / \mathbf{1 4}$ which shows a short-pitch helix formed in the $\mathrm{SmC}_{S} \mathrm{P}_{F}^{\text {hel }}$ in a higher-temperature range of SmC, a bilayer (antiferroelectric) structure in the lower-temperature range, and the coexistence of the two structures in between these two.

However, the uniform monodomain SS structure observed here appears to contradict the structure of a conventional SSFLC, where two different domains exist with the optical axis directed at $\pm \theta$ to the smectic layer normal. This phenomenon can be modeled by the surface anchoring energy. There are 
two terms in the total anchoring energy: (a) the in-plane term which accounts for the LC alignment [48], and (b) the out-of-plane term for stabilizing the SS structure. In a conventional calamitic SmC-like LC, the out-of-plane term in anchoring energy $F_{O}(\alpha)$ can be expanded in terms of $\sum_{n}(\sin \alpha)^{2 n}$, where $\alpha$ is the angle between the long molecular axis and the surface plane and $n$ is an integer. To the first approximation $(\alpha \ll 1)$ we can use the first (quadratic) term, i.e., $F_{O}(\alpha)=\gamma_{1} \sin ^{2} \alpha$. In calamitic SmC-like LCs this angle is a function of the tilt angle $\theta$ and the azimuthal angle $\varphi$ as $\alpha \cong \theta \sin \varphi \cdot \gamma_{1}$ is the coefficient for the out-of-plane anchoring energy term for the primary axis. The anchoring energy can be expressed as

$$
F_{O 1}(\varphi) \approx \gamma_{1} \alpha^{2} \approx \gamma_{1} \theta^{2} \sin ^{2} \varphi .
$$

This gives two minima in $F_{O 1}$, one for $\varphi=0^{\circ}$ and the second for $\varphi=180^{\circ}$. These correspond to the bistability of the two sets of domains, where the optical axes are directed at angles $\pm \theta$ to the smectic layer normal.

In the biaxial bent-core tilted smectics, the secondary (or biaxial) out-of-plane term must also be considered. This distinguishes between the energy for a set of molecules lying flat on the surface with those being perpendicular to the surface. Such a biaxial anchoring model was developed by Maclennan et al. [49] for the general case of tilted smectic layers. Their model satisfactorily explained the transition from the analog to the bistable electro-optic response in a ferroelectric bananashaped material.

In the simplified case of a bookshelf structure, the biaxial anchoring term is related to the angle between the secondary molecular axis and the surface plane (which is the same as the azimuthal angle, $\varphi) \cdot \gamma_{2}$ is the coefficient for the biaxial out-ofplane anchoring energy term, i.e., of the secondary axis. The biaxial anchoring energy can simply be written as

$$
F_{O 2}(\varphi) \approx \gamma_{2} \cos ^{2} \varphi \text {. }
$$

Therefore the total out-of-plane anchoring energy consists of both the primary and the secondary directors and is written as follows:

$$
F_{O}(\varphi)=F_{O 1}(\varphi)+F_{O 2}(\varphi)=\gamma_{1} \theta^{2} \sin ^{2} \varphi+\gamma_{2} \cos ^{2} \varphi .
$$

An excess of the anchoring energy for the helical structure can be calculated by an integration of the total out-of-plane anchoring energy [Eq. (5)] for a helical pitch as follows:

$$
\begin{aligned}
F_{O}(\text { hel }) & =\frac{1}{2 \pi} \int_{0}^{2 \pi}\left(\gamma_{1} \theta^{2} \sin ^{2} \varphi+\gamma_{2} \cos ^{2} \varphi\right) d \varphi \\
& =\left(\gamma_{1} \theta^{2}+\gamma_{2}\right) / 2 .
\end{aligned}
$$

$F_{O}(\varphi)$ being lower than $F_{O}(h e l)$ determines the stable state.

The first derivative of the out-of-plane anchoring energy when equated to zero gives the condition for the maximum or minimum of $F_{O}(\varphi)$ for the angle $\varphi$.

$$
F_{o}^{\prime}(\varphi)=\left(\gamma_{1} \theta^{2}-\gamma_{2}\right) \sin 2 \varphi=0 .
$$

The second derivative of $F_{O}(\varphi)$ is positive for values of $\varphi=$ $0^{\circ}$, and $180^{\circ}$ if the term within the parenthesis in Eq. (7) is positive, in which case $F_{O}(\varphi)$ is minimum for these two values of $\varphi$.
The term $\Delta_{O}=\left(\gamma_{1} \theta^{2}-\gamma_{2}\right)$ thus governs the minimum of the total anchoring energy $F_{O}$ for the preferred stable state. For sufficiently small values of $\Delta_{O} \approx 0$, the excess anchoring energy of all the four possible structures (helical, $\left.\varphi=0^{\circ}, 90^{\circ}, 180^{\circ}\right)$ is the same. Nevertheless the LC forms a helical structure which, unlike the remaining three, minimizes the excess electrostatic energy. The helical structure for the $\mathrm{SmC}_{S} \mathrm{P}_{F}^{\text {hel }}$ phase is thus realized over a temperature range of $\sim 90{ }^{\circ} \mathrm{C}-110^{\circ} \mathrm{C}$.

For positive $\Delta_{O}$ (which is always the case for a uniaxial LC, $\gamma_{2}=0$ ) there are two minimal energy states at $\varphi=0^{\circ}$, $180^{\circ}$ (as stated before), with the energy in two states equal; $F_{O}\left(0^{\circ}, 180^{\circ}\right)=\gamma_{2}$. These correspond to the conventional SSFLC structure with the two opposite $\pm \theta$ domains. However, for the biaxial case, $\gamma_{2}$ is finite; $\Delta_{O}$ becomes negative when the tilt angle $\theta$ reaches a reduced (or threshold) angle on cooling. The energy difference between the helical and $\varphi=90^{\circ}$ states is

$$
F_{O}(h e l)-F_{O}\left(90^{\circ}\right)=\frac{\gamma_{2}-\gamma_{1} \theta^{2}}{2}=-\frac{\Delta_{O}}{2} .
$$

Thus for negative $\Delta_{O}$, the domain for $\varphi=90^{\circ}$ is more stable than the helical state as its energy is the lower of the two. Hence under cooling, the tilted $\mathrm{SmC}_{S} \mathrm{P}_{F}^{\text {hel }}$ phase exhibits a structural transition from the helical to the surface-stabilized monodomain flat state, $\varphi=90^{\circ}$. In this state, the optical axis is directed along the layer normal.

For the case of LC comprised of uniaxial (calamitic) molecules (i.e., $\gamma_{2}=0$ and $\Delta_{\mathrm{O}}>0$ ) the minimum of the anchoring energy $F_{O}\left(0^{\circ}, 180^{\circ}\right)=0$ is achieved for the two domains $\left(\varphi=0^{\circ}, 180^{\circ}\right)$ of a conventional SSFLC. For orthogonal (SmA-like) biaxial phases, $\theta=0^{\circ}, \Delta_{\mathrm{O}}<0$, a minimum in the anchoring energy $F_{O}\left(90^{\circ}\right)=0$ is achieved. Thus $\varphi=90^{\circ}$ monodomain SS exists in an orthogonal smectic phase as well.

We can thus explain the transition occurring at $\sim 90{ }^{\circ} \mathrm{C}$ [Fig. 2(b)], by assuming that the coefficients $\gamma_{1}$ and $\gamma_{2}$ are temperature independent. In this case, $\Delta_{O}$ depends on the molecular tilt angle $\theta$ which decreases on cooling from $18^{\circ}$ down to $0^{\circ}$. This makes the anchoring energy factor $\Delta_{O}$ in favor of the monodomain SS structure $\left(\varphi_{0}=90^{\circ}\right)$. When the tilt angle reaches $\theta \approx 13^{\circ}$ (at a temperature of $\sim 90^{\circ} \mathrm{C}$ ) $\Delta_{\mathrm{O}}$ causes the helix to unwind and the transition leads to a SS monodomain texture.

\section{CONCLUSIONS}

Summarizing, we studied a smectic LC material constituted of the bent-core molecules using a range of techniques: electro-optics, polarizing microscopy, birefringence, and spontaneous polarization. Several interesting phenomena are observed. Strong evidence is given for an unusual and unexpected observation of a reversed temperature dependence of the tilt angle within the temperature range of the smectic phase. On cooling the sample LC aligned cell, the tilt angle first increases, then decreases, and finally it drops to zero. As a consequence, the phase transition from a tilted $\mathrm{SmC}_{A} \mathrm{P}_{A}$ phase to the orthogonal $\operatorname{SmAP}_{A}$ phase occurs under cooling. Reasons for the preferred existence of $\mathrm{SmAP}_{A}$ over $\mathrm{SmAP}_{F}$ 
are given. Prior to the transition having occurred, the tilted helical $\mathrm{SmC}_{S} \mathrm{P}_{F}^{\text {hel }}$ phase under cooling exhibits a transition from the helical state to the surface-stabilized helix-free state. The structure is proposed to be $\operatorname{SmC}_{A} \mathrm{P}_{A}$. Unlike the conventional two-domain SSFLCs, this SS structure is uniformly monodomain where the optical axis is directed parallel to the smectic layer normal. This structural transition is explained by considering the out-of-plane biaxial anchoring energy term.

\section{ACKNOWLEDGMENTS}

This work was partially supported by 13/US/I2866 from the Science Foundation Ireland as part of the US-Ireland Research and Development Partnership program jointly administered with the United States National Science Foundation under Grant No. NSF-DMR-1410649. One of the authors (J.K.V.) thanks Professor Satyendra Kumar for having successfully co-ordinated the NSF project on de Vries smectics.
[1] R. B. Meyer, L. Liebert, L. Strzelecki, and P. Keller, J. Phys. (Paris) 36, 69 (1975).

[2] A. Fukuda, Y. Takanishi, T. Isozaki, K. Ishikawa, and H. Takezoe, J. Mater. Chem. 4, 997 (1994).

[3] Y. Takanishi, A. Iida, N. Yadav, A. D. L. Chandani Perera, A. Fukuda, M. A. Osipov, and J. K. Vij, Phys. Rev. E 100, 010701(R) (2019).

[4] T. Niori, T. Sekine, J. Watanabe, T. Furukawa, and H. Takezoe, J. Mater. Chem. 6, 1231 (1996).

[5] K. J. K. Semmler, T. J. Dingemans, and E. T. Samulski, Liq. Cryst. 24, 799 (1998).

[6] A. Eremin, S. Diele, G. Pelzl, H. Nadasi, W. Weissflog, J. Salfetnikova, and H. Kresse, Phys. Rev. E 64, 051707 (2001).

[7] T. Sekine, T. Takanishi, T. Niori, J. Watanabe, and H. Takezoe, Jpn. J. Appl. Phys. 36, 6455 (1997).

[8] D. R. Link, G. Natale, R. Shao, J. E. Maclennan, N. A. Clark, E. Korblova, and D. M. Walba, Science 278, 1924 (1997).

[9] S. Sreenilayam, Y. P. Panarin, J. K. Vij, M. Osipov, A. Lehmann, and C. Tschierske, Phys. Rev. E 88, 012504 (2013).

[10] Y. P. Panarin, M. Nagaraj, J. K. Vij, C. Keith, and C. Tschierske, EPL 92, 26002 (2010).

[11] R. A. Reddy and C. Tschierske, J. Mater. Chem. 16, 907 (2006).

[12] H. Takezoe and Y. Takanishi, Jpn. J. Appl. Phys. 45, 597 (2006).

[13] T. Sekine, T. Niori, J. Watanabe, T. Furukawa, S.-W. Choi, and H. Takezoe, J. Mater. Chem. 7, 1307 (1997).

[14] J. Watanabe, T. Niori, T. Sekine, and H. Takezoe, Jpn. J. Appl. Phys. 37, L139 (1998).

[15] G. Pelzl, S. Diele, and W. Weissflog, Adv. Matter. 11, 707 (1999).

[16] G. Pelzl, S. Diele, A. Jákli, C. Lischka, I. Wirth, and W. Weissflog, Liq. Cryst. 26, 135 (1999).

[17] R. A. Reddy, C. Zhu, R. Shao, E. Korblova, T. Gong, Y. Shen, E. Garcia, M. A. Glaser, J. E. Maclennan, D. M. Walba, and N. A. Clark, Science 332, 72 (2011).

[18] D. Pociecha, M. Čepič, E. Gorecka, and J. Mieczkowski, Phys. Rev. Lett. 91, 185501 (2003).

[19] K. Gomola, L. Guo, D. Pociecha, F. Araoka, K. Ishikawa, and H. Takezoe, J. Mater. Chem. 20, 7944 (2010).

[20] N. Sebastian, S. Belau, A. Eremin, M. Alaasar, M. Prehm, and C. Tschierske, Phys. Chem. Chem. Phys. 19, 5895 (2017).

[21] Y. Shimbo, Y. Takanishi, K. Ishikawa, E. Gorecka, D. Pociecha, J. Mieczkowski, K. Gomola, and H. Takezoe, Jpn. J. Appl. Phys. 45, L282 (2006).

[22] M. Nagaraj, Y. P. Panarin, U. Manna, J. K. Vij, C. Keith, and C. Tschierske, Appl. Phys. Lett. 97, 213505 (2010).

[23] D. Krüerke and G. Heppke, Abstracts of the Workshop on Banana-Shaped Liquid Crystals: Chirality by Achiral Molecules, Berlin, 1997 (unpublished).
[24] A. Jákli, G. G. Nair, C. K. Lee, R. Sun, and L. C. Chien, Phys. Rev. E 63, 061710 (2001).

[25] D. M. Walba, E. Körblova, R. Shao, J. E. Maclennan, D R. Link, M. A. Glaser, and N. A. Clark. Science 288, 2181 (2000).

[26] M. Nakata, D. R. Link, F. Araoka, J. Thisayukta, Y. Takanishi, K. Ishikawa, J. Watanabe, and H. Takezoe, Liq. Cryst. 28, 1301 (2001).

[27] See Supplemental Material at http://link.aps.org/supplemental/ 10.1103/PhysRevResearch.2.013118 for a short history of the emergence of the phase structure of the investigated compound, XRD data, the POM textures and small and wide angle XRD scattering and a scheme explaining the polar phases of bent-core molecules are given.

[28] G. Heppke, A. Jákli, S. Rauch, and H. Sawade, Phys. Rev. E 60, 5575 (1999).

[29] M. Zennyoji, Y. Takanishi, K. Ishikawa, J. Thisayukta, J. Watanabe, and H. Takezoe, J. Mater. Chem. 9, 2775 (1999).

[30] G. Dantlgraber, A. Eremin, S. Diele, A. Hauser, H. Kresse, G. Pelzl, and C. Tschierske, Angew. Chem., Int. Ed. 41, 2408 (2002).

[31] L. M. Blinov, M. I. Barnik, E. S. Bustamante, G. Pelzl, and W. Weissflog, Phys. Rev. E 67, 021706 (2003).

[32] M. Nakata, R.-F. Shao, J. E. Maclennan, W. Weissflog, and N. A. Clark, Phys. Rev. Lett. 96, 067802 (2006).

[33] M. Nakata, D. Chen, R. Shao, E. Korblova, J. E. Maclennan, D. M. Walba, and N. A. Clark, Phys. Rev. E 85, 031704 (2012).

[34] S. P. Sreenilayam, Yu. P. Panarin, J. K. Vij, V. P. Panov, A. Lehmann, M. Poppe, M. Prehm, and C. Tschierske, Nat. Commun. 7, 11369 (2016), and Supplementary Information therein, Fig. 4.

[35] A. A. S. Green, M. R. Tuchband, R. Shao, Y. Shen, R. Visvanathan, A. E. Duncan, A. Lehmann, C. Tschierske, E. D. Carlson, E. Guzman, M. Kolber, D. M. Walba, C. S. Park, M. A. Glaser, J. E. Maclennan, and N. A. Clark, Phys. Rev. Lett. 122, 107801 (2019).

[36] M. Alaasar, M. Prehm, M. Nagaraj, J. K. Vij, and C. Tschierske, Adv. Mater. 25, 2186 (2013).

[37] M. Alaasar, M. Prehm, M. Poppe, M. Nagaraj, J. K. Vij, and C. Tschierske, Soft Matter 10, 5003 (2014); J. K. Vij, Yu. P. Panarin, S. P. Sreenilayam, M. Alaasar, and C. Tschierske, Phys. Rev. Mater. 3, 045603 (2019).

[38] K. L. Sandhya, Yu. P. Panarin, V. P. Panov, J. K. Vij, and R. Dabrowski, Eur. Phys. J. E 27, 397 (2008).

[39] W. Weissflog, C. Lischka, S. Diele, I. Wirth, and G. Pelzl, Liq. Cryst. 27, 43 (2000). 
[40] O. E. Panarina, Yu. P. Panarin, F. Antonelli, J. K. Vij, M. Reihmann, and G. Galli, J. Mater. Chem. 16, 842 (2006).

[41] S. Sreenilayam, Yu. P. Panarin, J. K. Vij, A. Lehmann, and C. Tschierske, Mol. Cryst. Liq. Cryst. 610, 116 (2015).

[42] S. A. Pikin and V. L. Indenbom, Ferroelectrics 20, 151 (1978).

[43] L. A. Beresnev, V. G. Chigrinov, D. I. Dergachev, E. P. Poshidaev, J. Fünfschilling, and M. Schadt, Liq. Cryst. 5, 1171 (1989).

[44] Yu. P. Panarin, S. P. Sreenilayam, J. K. Vij, A. Lehmann, and C. Tschierske, J. Mater. Chem. C 5, 12585 (2017).
[45] D. Pociecha, E. Gorecka, M. Čepič, N. Vaupotič, K. Gomola, and J. Mieczkowski, Phys. Rev. E 72, 060701(R) (2005).

[46] M. W. Schröder, S. Diele, N. Pancenko, W. Weissflog, and G. Pelzl, J. Mater. Chem. 12, 1331 (2002).

[47] Y. Lansac, P. K. Maiti, N. A. Clark, and M. A. Glaser, Phys. Rev. E 67, 011703 (2003).

[48] Yu. P. Panarin, S. T. Mac Lughadha, and J. K. Vij, Phys. Rev. E 52, R17 (1995).

[49] J. E. Maclennan, N. A. Clark, and D. M. Walba, Phys. Rev. E 64, 031706 (2001). 